\title{
EVOCACIÓN POR EL 350 ANIVERSARIO DE VICO
}

\author{
Alain Pons \\ (Universidad de París, Nanterre)
}

RESUMEN: Una reflexión acerca de lo que ha significado para la labor filosófica del Autor la obra y el pensamiento de Vico. El Autor se pregunta por las circunstancias que le han llevado a dedicar a Vico una parte importante de su actividad investigadora y docente. No se trata de hacer una autobiografía intelectual sino solo de evocar, a partir de su propia experiencia francesa, su participación en la producción internacional de filósofos, filólogos e historiadores estudiosos del pensamiento y de la obra de Vico.

Palabras Clave: Vico, $350^{\circ}$ Aniversario, Siglo de las Luces, razón ilustrada, «Internacional Viquiana», A. Pons.

Evocation in occasion of the $350^{\text {th }}$ Anniversary of Vico

ABSTRACT: This is a reflection on what the work and thought of Vico has meant for the philosophical work of the Author. The author wonders which are the circumstances that led him to devote an important part of his own research and teaching duties to Vico. The point is not to offer any intellectual autobiography, but only to evoke, from his own French experience, his participation in the international production of philosophers, philologists and historians who study Vico's thought and work.

KeYwords: Vico, $350^{\text {th }}$ Anniversary, Enlightenment, enlightened reason, «Vichian International», A. Pons.

\section{Evocazione in occasione del $350^{\circ}$ anniversario di Vico}

RIASSUNTO: Si intende offrire una riflessione su ciò che l'opera e il pensiero di Vico hanno significato per il lavoro filosofico dell'Autore. L'Autore si interroga circa le circostanze che lo hanno portato a dedicare una parte importante delle sue attività di ricerca e di insegnamento a Vico. Non si tratta di realizzare un'autobiografia intellettuale ma solo di rievocare, a partire dalla sua esperienza francese, la sua partecipazione alla produzione internazionale di filosofi, filologi e storici che studiano il pensiero e l'opera di Vico.

Parole Chiave: Vico, $350^{\circ}$ Anniversario, Illuminismo, ragione illuminata, «Internationale Vichiana», A. Pons.

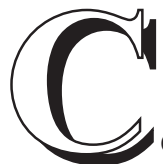

elebro la acertada iniciativa del profesor Sevilla que ha permitido a quienes, desde hace algunos años, se han interesado en Vico y en su obra reflexionar sobre lo que el filósofo napolitano ha significado en su labor filosófica. En lugar de intentar sintetizar en algunas páginas el trabajo que haya podido realizar en este

Este artículo responde a una invitación expresa por parte de la Dirección de la Revista para este volumen especial por el $350^{\circ}$ Aniversario del nacimiento de G. Vico, habiendo superado los criterios de valoración y del proceso de aceptación. 
ámbito, me gustaría en primer lugar preguntarme por las circunstancias que me han llevado a dedicar a este autor una parte importante de mi actividad investigadora y docente. No se tratará de hacer una autobiografía intelectual sino solo de evocar, a partir de mi propia experiencia francesa, mi participación en los trabajos de lo que llamaré la "Internacional Viquiana", es decir: el conjunto de filósofos, filólogos, historiadores, de cualquier nacionalidad, de cualquier campo intelectual, que han estudiado a Vico desde hace más de cincuenta años.

Interesarse en el autor de la Scienza nuova para un joven filósofo francés en los años Sesenta no era lo habitual. Desde Michelet se conocía su nombre en Francia, pero su obra no había sido asimilada en el sistema universitario francés, como lo había sido en Italia, al mismo nivel de aquellos autores importantes sin cuyo conocimiento la enseñanza de la filosofía no podía estar completa. Así pues, para mí fue el interés por la "filosofía de la historia", puesta de moda en Francia por la traducción de Hegel, en particular, y los consejos de mi maestro Raymond Aron, lo que atrajo mi atención por Vico y me llevó a solicitar una beca de estudios para el "Istituto italiano per gli studi storici", en Nápoles, que por entonces estaba dirigido por el gran historiador Federico Chabod. En ese Palazzo Filomarino, donde la presencia intelectual de Benedetto Croce estaba aún presente, yo trabajaba en mi aprendizaje de la lengua italiana y comenzaba a leer a Vico, lo que me llevó a visitar a Fausto Nicolini. De regreso a Francia, comencé en la Universidad de París-Nanterre un curso de historia de la filosofía política centrado principalmente en el pensamiento francés del Siglo Dieciocho.

En 1961, publiqué en la revista Les Études philosophiques un estudio sobre «Nature et histoire chez Vico» [Naturaleza e historia en Vico], seguido en los años siguientes de algunos artículos dedicados al mismo autor. En 1968, con ocasión del tricentenario del nacimiento de Vico, entré en contacto con Giorgio Tagliacozzo, a quien quiero rendir homenaje por la importante contribución que ha aportado a los estudios viquianos, en Estados Unidos y en Europa, por sus numerosas iniciativas (publicación de obras colectivas, organización de congresos y conferencias). Es conocido el papel desempeñado por el "Centro di studi vichiani" de Nápoles, fundado por Pietro Piovani, con su Bollettino, y más tarde, en Estados Unidos, por el "Institute for Vico studies", dirigido por Giorgio Tagliacozzo y Donald Phillip Verene, y, en España, por los Cuadernos sobre Vico, fundados y dirigidos por José M. Sevilla. Así es como la multiplicación de manifestaciones "viquianas", conferencias, coloquios, congresos, supuso para mí la oportunidad de encontrar a muchos colegas y de entablar con algunos de ellos vínculos de amistad intelectual y personal, con cuyo contacto mi reflexión sobre Vico se ha enriquecido constantemente. No los nombraré, son demasiado numerosos, muchos han desaparecido, pero me complace comprobar que incluso en Francia, a una escala aún modesta, el interés por Vico ha aumentado. 
Ha sido en esta atmósfera intelectual donde he llevado a cabo mis propios trabajos. No voy a enumerar las diferentes publicaciones en las que he continuado mi reflexión sobre el filósofo napolitano; sin embargo, quiero insistir en la conexión estrecha que ha unido en mí el trabajo de traducción y el de exégesis y comentarista. En 1981 he traducido del italiano la Vita di Giambattista Vico scritta da se medesimo, con una selección de "Cartas", y del latín, el De nostri temporis studiorum ratione. He retomado la traducción del De ratione en 2010, a partir de un texto preparado por Andrea Battistini. Fue en 2001 cuando aporté una traducción de la Scienza nuova de 1744. La satisfacción que ha sido para mí traducir los grandes textos italianos (el Libro del Cortegiano de Baldassar Castiglione, el Galateo ovvero de' costumi de Della Casa, los Ricordi de Guicciardini) ha ido acompañada, en el caso de la Scienza nuova, de la convicción de que, en el caso de Vico sobre todo, el trabajo de interpretación debe apoyarse esencialmente en una reflexión en relación con su estilo y vocabulario, habida cuenta de que en él la filología y la filosofía están esencialmente ligadas. De este modo las dificultades debidas a la traducción de la Scienza nuova me han ayudado enormemente en mi lectura filosófica.

He expuesto las grandes líneas de esta interpretación en una obra mía aparecida en 2015, Vie et mort des nations. Lecture de la Science nouvelle de Giambattista Vico, pero no se trata aquí de presentar este trabajo. Quisiera solo decir algunas palabras sobre una cuestión de la que, no habiendo sido hasta ahora ignorada, me parece sin embargo que queda todavía mucho por decir: el lugar que sería preciso asignar a Vico en su siglo, es decir, en lo que se conoce como «siglo de las Luces». Los historiadores de las ideas han planteado esta cuestión a menudo, pero yo me siento en particular interesado por ella en la medida en que una parte de mis trabajos ha versado sobre algunos autores franceses del Siglo Dieciocho especialmente representativos de este «espíritu de las Luces»: publicación de una selección de artículos de la Encyclopédie dirigida por Diderot y d'Alembert, edición del Dictionnaire philosophique de Voltaire y la del Esquisse d'un tableau historique des progrès de l'esprit humain de Condorcet.

¿Podríamos, en efecto, preguntarnos lo que Vico habría pensado de la obra monumental de Diderot y d'Alembert, sabiendo que lamenta en su carta al Padre de Vitry, en 1726, que los hombres de letras de su época «sientan la necesidad de acomodarse al espíritu del siglo, más ávido este de resumir lo que otros han aprendido que de profundizar yendo más lejos. De este modo, sintiéndose obligados a trabajar en diccionarios, bibliotecas, compendios», exactamente como lo hicieron los últimos intelectuales de la Grecia decadente? Y, otro ejemplo, ¿cuál habría sido la reacción del autor del De nostri temporis studiorum ratione al leer, en esta misma Encyclopédie, cuya publicación comienza en 1751, el artículo Collège, en el cual d'Alembert habla de los estudios que deberían seguir los jóvenes: en filosofía, la lógica se limitaría a algunas líneas; en metafísica, a un resumen de Locke; la moral 
puramente filosófica, a las obras de Séneca y de Epicteto; la moral cristiana, al sermón de Jesucristo en la montaña; la física, a lo empírico y a la geometría? ¿Qué habría pensado de los ataques furibundos de Voltaire al judaísmo y al cristianismo, o de la visión de la historia de Condorcet al dibujar un «retrato de la especie humana, liberada de todas sus cadenas, sustraída del imperio del azar como del imperio de los enemigos del progreso»?

La respuesta a estas cuestiones es fácil. Vico habría reconocido en las obras de d'Alembert, Diderot, Voltaire, Helvétius, d'Holbach, si hubiese podido leerlas, la influencia de esos filósofos antiguos y modernos puestos en tela de juicio a lo largo de su Scienza nuova, la de los epicúreos antiguos y sobre todo modernos que él cita en numerosas ocasiones, Maquiavelo, Hobbes, Spinoza, Locke, Bayle, y también, por razones diferentes, la del racionalismo de Descartes. Pero ¿es esto una razón suficiente para hacer de él un «anti-moderno» por excelencia, y, más aún, el primer representante de la «anti-Ilustración», ya se trate de las Lumières francesas, del Enlightenment inglés, de la Aufklärung alemana o del Illuminismo italiano?

De hecho, la dificultad para ubicar a Vico en su siglo viene de la manera restrictiva y simplificadora con la que se define la Ilustración. Si solo se ve en esta la prefiguración de las posiciones radicales del ateísmo, del materialismo, del igualitarismo político y económico, que desembocarán en la Revolución Francesa, o, por lo menos, un espíritu general de libertad crítica en la manera de hablar de lo que hasta entonces había estado protegido por las tradiciones religiosas, morales y políticas, está claro que Vico no es un hombre de la Ilustración. Pero ¿por qué dar a la palabra "Ilustración" un sentido tan restringido? No olvidemos la manera en la que Kant la definirá: «¡Sapere aude! ¡Ten el coraje de servirte de tu propio entendimiento! He aquí la divisa de la Ilustración (Aufklärung)». Si este es el espíritu profundo de la Ilustración, ¿no es imposible no reconocerlo en Vico cuando dedica su primera Scienza nuova, en 1725, a

«las academias de Europa, que, en esta época ilustrada (età illuminata) en la que no solo las fábulas y las tradiciones vulgares de la historia pagana, sino toda autoridad, sea cual fuere, de los más célebres filósofos, se somete a la crítica de una razón severa»?

Encontramos aquí los términos propios del vocabulario de la Ilustración: "época ilustrada", "crítica", "razón".

La audacia, el coraje intelectual de Vico, reside en querer servirse de su propio entendimiento para fundar una ciencia que no existe aún, una «ciencia nueva». Desde 1720, escribe que había realizado un esfuerzo por elaborar «un sistema de la civilización, de las repúblicas, de las leyes, de la poesía, de la historia, en una palabra de toda la humanidad, y esto en consonancia con una filología razonada». Y, al principio de la Ciencia nueva de 1744, Vico declara que en su obra: 
«la filosofía tiene la tarea de examinar a la filología, es decir la doctrina de todo lo que depende del arbitrio humano, como es el caso de todas las historias de las lenguas, de las costumbres y de los hechos de los pueblos en la paz como en la guerra $[. .$.$] y la reduce a la forma de$ una ciencia, descubriendo en ella el propósito de una historia ideal eterna que siguen en el tiempo las historias de todas las naciones». $(S N 44, \S 7)$

La ambición de su «ciencia» consiste, pues, en examinar filosóficamente el campo de los asuntos humanos, cuyo estudio corresponde hoy a las ciencias llamadas «humanas». Este vasto campo el Siglo de las Luces no lo ha ignorado, lo ha explorado tanto en Francia como en Inglaterra, en Alemania, en Italia y en otros países. Lo demuestran, desde luego, l'Encyclopédie, l'Essai sur les mours de Voltaire, el Esprit des Lois de Montesquieu, el Contrat social de Rousseau, el Tableau historique de Condorcet, entre las obras más conocidas, pero también todas aquellas que tratan del derecho y de su historia, de la economía y del comercio, del lenguaje, de las lenguas, de la moral, de la educación, de las instituciones políticas, de la historia de los pueblos civilizados, de las sociedades primitivas conocidas por los relatos de los viajeros.

Lo que Vico hará será someter este material, que proviene de lo que él llama la «filología», a un análisis «filosófico» confiriéndole una unidad y una perspectiva coherente. Se trata de una filosofía del espíritu humano, que no se corresponde con la del racionalismo cartesiano, y que pone de relieve el poder creador de la imaginación, gracias a la cual el hombre construye el mundo, inventa sus dioses y sus propias instituciones humanas. La ciencia viquiana no es sino la tentativa grandiosa de dar cuenta de la manera en la que los hombres han construido, en el tiempo, su humanidad. Ella se apoya en una fe religiosa cristiana manifiesta, excluyendo el materialismo y el deísmo; todo el sistema de Vico supone la existencia de una providencia, pero él utiliza los textos judaicos y cristianos, principalmente la Biblia, no de una manera apologética, sino como documentos históricos que permiten comprender cómo, a partir de una naturaleza caída, los hombres han conquistado su plena humanidad. Vico se apoya por otra parte en el conocimiento de las instituciones griegas y romanas y de la mitología que él interpreta de un modo muy original. Reconoce, pues, la existencia de un «sentido común» donde lo que él llama las «costumbres» constituyen sus manifestaciones evolucionando en el tiempo. Se trata de prácticas religiosas, matrimonios, modos de inhumación, de reglas jurídicas, de instituciones sociales y políticas, estudiadas en su aparición y su devenir. Estas costumbres no son arbitrarias, y lo que Vico denomina «filología» lo llamaríamos hoy "ciencias humanas", es decir la historia, la sociología, la antropología, la lingüística, le permite a Vico conocer su aparición y sus transfor- 
maciones en el curso (corso) que siguen las naciones, en la medida que las naciones son las formas históricas en las que se resuelve el paso hacia su plena realización de la humanidad caída .

La audacia "ilustrada" de Vico es haber querido acoger en una ciencia nueva los datos de la religión, de la filosofía y de la filología en un vasto sistema que supone tener en cuenta la dimensión temporal de la humanidad en la totalidad de sus manifestaciones. En este sentido su "ciencia nueva" es mucho más que una "filosofía de la historia", si entendemos por historia el relato comentado de los hechos humanos. Su «tentativa», como la definía él mismo, es ciertamente una de las manifestaciones más originales, más audaces, del espíritu de la Ilustración en su máxima expresión.

[Traducción del francés por Fernanda Pérez Alors]

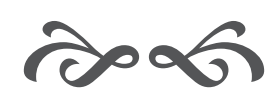

